\title{
Optimum Goodput-Oriented Power Allocation Policy for BIC-OFDM Packet Transmissions
}

\author{
I. Stupia $\nmid$, L. Vandendorpe $\ddagger$ J. Louveaux $\ddagger$, F. Giannetti†, V. Lottici $\dagger$, N.A. D’Andrea $\dagger$ \\ $\dagger$ Department of Information Engineering, University of Pisa, \\ Via G. Caruso, 16 - I-56122 Pisa, Italy \\ $\ddagger$ Communications and Remote Sensing Lab, Université Catholique de Louvain, \\ Place du Levant, 2 - B-1348 Louvain La Neuve, Belgium \\ \{ivan.stupia, filippo.giannetti, vincenzo.lottici,aldo.dandrea $\} @$ iet.unipi.it, \\ \{luc.vandendorpe, jerome.louveaux\}@uclouvain.be
}

\begin{abstract}
In this contribution we study the optimal power allocation scheme for a coded multicarrier transmission. Specifically, we focus on a bit interleaved coded modulation (BICM) packet transmission implemented with Orthogonal Frequency Division Multiplexing (OFDM) and in the presence of automatic repeat request $(A R Q)$ protocol. Based on a simple lower-bound of the rate of information bits received without any error, the so called goodput, we develop a power allocation strategy among the different subcarriers so that the system goodput performance metric is maximized. We also demonstrate the optimality of the solution for the proposed objective function under the given power constraint. The effectiveness of the power allocation policy is numerically testified for BIC-OFDM transmission in the context of typical indoor environment.
\end{abstract}

\section{INTRODUCTION}

At the high data rates typically required to support multimedia services, harsh multipath propagation conditions are typically experienced in both urban outdoor and indoor terrestrial scenarios, thereby making the design of an efficient and reliable transmission scheme a particularly demanding task. A viable answer to this need consists in a cross-layer design approach where a mix of up-to-date efficient techniques for modulation, channel coding and link adaptation are properly combined. In the context of high data rate transmissions over wireless frequency selective channels, one of the most efficient modulation formats is represented by multicarrier (MC) techniques. In the form of orthogonal frequency division multiplexing (OFDM), MC schemes have been embedded in several standards such as Wi-Fi WLAN IEEE $802.11 \mathrm{a} / \mathrm{g} / \mathrm{h}$, Wi-Max broadband wireless access IEEE 802.16, and digital audio and video broadcasting (DAB and DVB). In order to further increase the system robustness against the troubles arising from the wireless propagation channels, an efficient modulation scheme has to be combined, however, with an as much as powerful channel coding technique. This is the case of BICM, which was proposed in 1992 by Zehavi as a pragmatic coding scheme for bandwidth-efficient communications [1]. This is based on the insertion of a bit-interleaver between the channel encoder and the modulator in order to increase the diversity order. Later, a theoretical foundation for BICM was given by Caire, Taricco and Biglieri [2]. Different methods to evaluate the performances of BICM systems have been proposed. Most of them are based on the union bound and the expurgation technique proposed in [2]. Recently, in [3], thanks to the binary-input output-symmetric (BIOS) nature of the channel, a simple yet accurate computation of the Pair-Wise Error Probability (PEP) based on the saddlepoint approximation has been provided. In [9] this approach has been extended to the MIMO-BICM systems. The superior code diversity and the design flexibility of BICM have motivated many common wireless applications. For instance IEEE 802.11 WLAN is an application of BICM which is implemented with OFDM.

The goal of a cross-layer design is fully achieved on condition that efficient link adaptation schemes be properly employed as well. The idea is to optimize the overall system performance under the constraint of fixed radio resources. To this end, the water-filling policy [11] has gained a considerable interest to power and bit resource allocation across the subcarriers of a OFDM-based system. In [4], through the extension of the conventional BICM-OFDM Pair-Wise Error Probability (PEP) analysis, a bit and power allocation algorithm has been proposed to improve the total bit-rate. However, in some WLAN-based applications, only error-free packets are kept by the receiver, while the others are retransmitted through an automatic repeat request (ARQ) retransmission mechanism. Therefore, an optimized allocation strategy has necessarily to maximize over the available resources the number of transmitted bits in the error-free packet by unit of time, or goodput for short. Based on the above baseline and differently from the works published so far in the literature, the aim of this paper is to present a power allocation strategy that aims at improving the goodput achievable in a packet-based BICMOFDM sytems. The theoretical foundation of the allocation algorithms consists in a simplified PEP analysis. After delineating in Sect. II the BICM-OFDM model, Sect. III will be devoted to the evaluation of a simple upper bound of the goodput metric. Simulation results will be provided in Sect. IV for a typical WLAN scenario, followed some concluding remarks in Sect. V.

Notations: Matrices are in upper case bold (e.g., W), while column vectors are in lower case bold with an underscore (e.g., $\underline{\mathbf{w}}$ ). Also, $(\cdot)^{T}$ is used to denote the transpose operation and $\mathbf{D}(\cdot)$ is the diagonalization operator which converts an $N$ - 
dimensional vector into an $N \times N$ diagonal matrix. Finally, $\mathrm{E}_{V}\{\cdot\}$ is the statistical expectation over the random variable (RV) $V, \underline{1}$ denotes the vector with all entries one and $p(V \mid E)$ is the probability density function (p.d.f.) of $V$ conditioned on the event $E$.

\section{System Description And Performance Analysis}

A. System Model

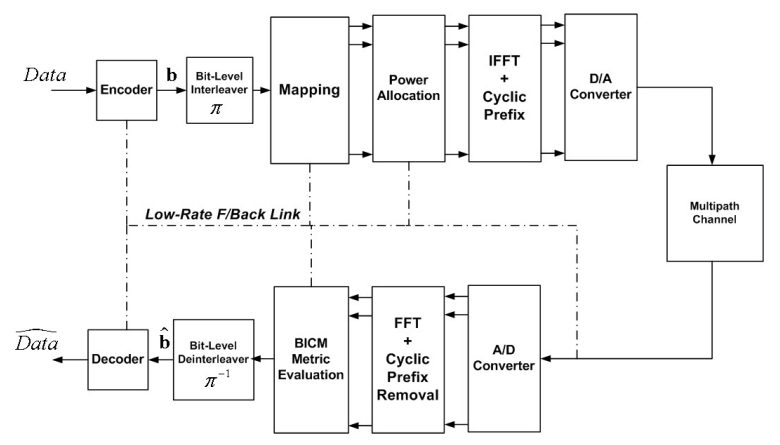

Fig. 1. System Model.

With reference to the block diagram depicted in fig.1, a packet of $N_{b}$ information bits is transmitted through a frame of $L$ OFDM blocks. Denoting the bit rate as $R_{b} \triangleq 1 / T_{b}$, where $T_{b}$ is the bit interval, the packet (or the OFDM frame) duration turns out $T_{p} \triangleq N_{b} T_{b}$. The information bits are first encoded using a convolutional code with rate $R$ and free distance $d_{\text {free }}$. The total number of coded binary symbols that are generated by encoding a packet of $N_{b}$ information bits is thus $N_{c} \triangleq$ $N_{b} / R$. The total available bandwidth is split into $N$ subcarriers and the number of bits allocated to the modulation symbol transmitted on the $n$th subcarrier is $m^{(n)}$. The latter parameter is independent of the OFDM block and is kept constant during the whole packet transmission.

A bit-interleaver (П) randomly maps the generic coded binary symbol $b_{k}$ into one of the bits carried by the symbols of the OFDM subcarriers according to the notation shown below

$$
b_{k} \rightarrow c_{\Pi(k)}
$$

where $\Pi(k) \triangleq\left\{l_{k}, n_{k}, i_{k}\right\}$ is the interleaver operation that maps the index $k$ of the coded binary symbol into a set of three coordinates: $l_{k}$ denotes the position of the OFDM block within the frame, $n_{k}$ denotes the position of the subcarrier , and $i_{k}$ denotes the position of the bit within the modulation symbol allocated into such subcarrier. The interleaver is assumed to be fully random, so that the probability of mapping the generic coded binary symbol $b_{k}$ (taken out of the available $N_{c}$ ) into the generic bit $c_{l, n, i}$, with $l=1, \cdots, L, n=1, \cdots, N$, and $i=1, \cdots, m^{(n)}$, is

$$
\operatorname{Pr}\left\{b_{k} \rightarrow c_{l, n, i}\right\} \triangleq \frac{1}{N_{c}},
$$

The interleaved bits are then Gray mapped into QAM symbols. The $M^{(n)}$-QAM signal set used in the subcarrier $n$ is defined as $\chi^{(n)} \triangleq\left\{\xi_{1}^{(n)}, \cdots, \xi_{M^{(n)}}^{(n)}\right\}$, with $M^{(n)} \triangleq$ $2^{m^{(n)}}$, and its symbols are suitably normalized such that $\sum_{\nu=1}^{M^{(n)}}\left|\xi_{\nu}^{(n)}\right|^{2} / M^{(n)}=1$. The generic data-bearing symbol $x_{l}^{(n)}$, belonging to the set $\chi^{(n)}$, is sent on the subcarrier $n$ during the OFDM block $l$, and $\mathrm{E}_{x_{l}^{(n)}}\left\{\left|x_{l}^{(n)}\right|^{2}\right\}=1$. The average transmit power allocated to the generic subcarrier $n$ is $P^{(n)} \triangleq p^{(n)} S$, where $p^{(n)}$ is a power coefficient $0 \leq p^{(n)} \leq 1$, so that the constraint on the average transmit power per subcarrier is expressed by

$$
\bar{P} \triangleq \frac{\sum_{n=1}^{N} P^{(n)}}{N} \leq S .
$$

where $S$ is the maximum value of the average power which can be allocated to every subcarrier.

The data symbols are frequency mapped to the $N$ available subcarriers using an Inverse Discrete Fourier Transform (IDFT) unit, which provides one sample every $T_{s}$ seconds. A conventional cyclic prefix (CP), whose length is $N_{c p}$ samples, is inserted at the beginning of each IDFT output block to maintain the subcarriers orthogonal with each others and avoid interference between successive symbols. The resulting OFDM signal experiences a frequency selective fading channel, and we will assume the channel stationarity during the whole packet (or OFDM frame) duration.

At the receiver side the samples are collected into blocks of size $N+N_{c p}$. After CP removal, they are transformed by a DFT unit of size $N$. Let us focus now on the transmission of the coded binary symbol $b_{k}$. According to the notations outlined above, after interleaving and modulation mapping, the bit is included into the QAM symbol $x_{l_{k}}^{\left(n_{k}\right)}$. The expression of the sample relevant to subcarrier $n_{k}$ in the OFDM block $l_{k}$, at the output of the DFT unit of the receiver is

$$
z_{l_{k}}^{\left(n_{k}\right)}=A^{\left(n_{k}\right)} x_{l_{k}}^{\left(n_{k}\right)}+w_{l_{k}}^{\left(n_{k}\right)}
$$

where

$$
A^{\left(n_{k}\right)} \triangleq \sqrt{P^{\left(n_{k}\right)}} H^{\left(n_{k}\right)}=\sqrt{p^{\left(n_{k}\right)} S} H^{\left(n_{k}\right)}
$$

and $w_{l_{k}}^{\left(n_{k}\right)}$ is a zero-mean unit-variance complex Gaussian RV representing the channel noise contribution. For the sake of simplicity, we resort to the following vectorial notation which includes all the subcarrier samples relevant to OFDM block $l$

$$
\underline{\mathbf{z}}_{l_{k}}=\mathbf{A} \underline{\mathbf{x}}_{l_{k}}+\underline{\mathbf{w}}_{l_{k}}
$$

where $\mathbf{A}$ is a complex $N$-dimensional diagonal matrix defined as $\mathbf{A} \triangleq \mathbf{D}\left(A^{(1)}, \cdots, A^{(N)}\right)$, while the symbol and the noise vectors are $\underline{\mathbf{x}}_{l_{k}} \triangleq\left[x_{l_{k}}^{(1)}, \cdots, x_{l_{k}}^{(N)}\right]^{T}$, and $\underline{\mathbf{w}}_{l_{k}} \triangleq$ $\left[w_{l_{k}}^{(1)}, \cdots, w_{l_{k}}^{(N)}\right]^{T}$, respectively.

\section{B. Goodput Evaluation}

In the current section we provide a theoretical characterization of the goodput (GP) of the system outlined above. Let first consider two distinct codewords, i.e., two sequences of coded binary symbols which originate from the same state and merge after $d$ trellis steps, and let denote them as $d$ dimensional arrays $\underline{\mathbf{b}}$ and $\underline{\mathbf{b}}^{\prime}$, whose $k$ th element is $b_{k}$ and $b_{k}^{\prime}$, respectively. We also denote with $\underline{\hat{\mathbf{b}}}$ a codeword at the 
decoder output. The first step of our analysis will be evaluate the pairwise error probability (PEP), defined as

$$
P E P \triangleq \operatorname{Pr}\left\{\underline{\hat{\mathbf{b}}}=\underline{\mathbf{b}^{\prime}} \mid \underline{\mathbf{b}}, \mathbf{A}\right\} .
$$

In case of ideal channel state information (CSI), i.e., assuming that the actual value of the random channel gain $\mathbf{A}$ is known, the BICM log-likelihood metric for the $k$ th coded binary symbol at the decoder input can expressed as

$$
\Lambda_{k}=\log \frac{\sum_{\tilde{x} \in \chi_{b_{k}^{\prime}}^{\left(i_{k}, n_{k}\right)}} p\left(z_{l_{k}}^{\left(n_{k}\right)} \mid x_{l_{k}}^{\left(n_{k}\right)}=\tilde{x}, \mathbf{A}\right)}{\sum_{\tilde{x} \in \chi_{b_{k}}^{\left(i_{k}, n_{k}\right)}} p\left(z_{l_{k}}^{\left(n_{k}\right)} \mid x_{l_{k}}^{\left(n_{k}\right)}=\tilde{x}, \mathbf{A}\right)}
$$

where $\chi_{a}^{(i, n)}$ represents the subset of all the M-QAM symbols $\xi_{\nu}^{(n)} \in \chi^{(n)}$ whose $i$ th bit is equal to $a$.

Under the assumption of ideal interleaving, the BICM subcarriers behave as a memoryless BIOS channels and the PEP can be computed as the tail probability [3]

$$
P E P=\operatorname{Pr}\left\{\sum_{k=1}^{d} \Lambda_{k}>0\right\}=\operatorname{Pr}\{\Theta>0\},
$$

where we defined $\Theta \triangleq \sum_{k=1}^{d} \Lambda_{k}$. Unfortunately, the computation of (9) by the p.d.f. of $\Theta$ is too involved.

Exploiting the fact that the log-likelihood ratios $\Lambda_{k}$ are i.i.d. RVs, a simple method to estimate the PEP (9) is the Bhattacharyya Bound, given by

$$
P E P \leq\left[M_{\Lambda}(\hat{s})\right]^{d}
$$

where $M_{\Lambda}(s) \triangleq \mathrm{E}_{\Lambda_{k}}\left\{\exp \left(s \Lambda_{k}\right)\right\}$ is the Moment Generating Function (MGF) of the RV $\Lambda_{k}$ and $\hat{s}$ is the saddlepoint. We remark that, for BIOS channels $\hat{s}=1 / 2$ and the Bhattacharyya Bound coincides thus with the Chernoff Bound [3]. As demonstrated in [10] the MGF can be approximated as

$$
M_{\Lambda}(s) \simeq \sum_{n_{k}=1}^{N} \operatorname{Pr}\left(n_{k}\right) \exp \left(-\left|A^{\left(n_{k}\right)}\right|^{2} d_{\min }^{\left(n_{k}\right)^{2}}\left(s-s^{2}\right)\right)
$$

where

$$
\operatorname{Pr}(n) \triangleq \frac{m^{(n)}}{\sum_{j=1}^{N} m^{(j)}}
$$

is the probability that a codeword bit is sent through the $n$th subcarrier in the case of ideal random interleaving and $d_{\min }^{\left(n_{k}\right)}$ denotes the minimum Euclidean distance between the symbols in the complete QAM set $\chi^{\left(n_{k}\right)}$ associated with the subcarrier $n_{k}$. Let us remark that, for a given channel status A, the PEP doesn't depend on the codewords (i.e., on the data sequences) $\underline{\mathbf{b}}$ and $\underline{\mathbf{b}}^{\prime}$, but only on their Hamming distance $d$, so that we can adopt the following notation: $P E P=\operatorname{Pr}(d \mid \mathbf{A})$. Then, by using the union bound, the packet error rate (PER) $P_{u}(\mathbf{A})$ is bounded by

$$
P_{u}(\mathbf{A}) \leq \sum_{d=d_{\text {free }}}^{N_{c}} \omega(d) \operatorname{Pr}(d \mid \mathbf{A})
$$

where $\omega(d)$ is the weight of all the error events having Hamming distance $d$, and $d_{\text {free }}$ is the minimum distance between two codewords. Due to the complexity of the problem, in order to provide a manageable expression of the goodput, we will use the Battacharyya bound. Further, for the sake simplicity, let us refer to the Battacharyya PEP upper bound simply as PEP. Replacing the PEP (10) in (13), we get

$P_{u}(\mathbf{A}) \leq \sum_{d=d_{\text {free }}}^{N_{c}} \omega(d)\left[\sum_{n=1}^{N} \operatorname{Pr}(n) \exp \left(\frac{-\left|A^{(n)}\right|^{2} d_{\text {min }}^{(n)}{ }^{2}}{4}\right)\right]_{(14)}^{d}$

The goodput (GP) is defined as the number of data bits delivered in error-free packets per unit of time. Assuming the transmission and re-transmission of the packets controlled by the selective-repeat (SR) ARQ scheme described in [7] and taking the OFDM symbol interval as the time reference, we can express the goodput as

$$
G P=R \sum_{n=1}^{N} m^{(n)} \cdot\left[1-P_{u}(\mathbf{A})\right] .
$$

\section{Optimal Power Allocation}

This section describes the power allocation strategy to improve the system goodput performance.

\section{A. Problem Formulation}

Let us note that the only term in the goodput expression (15) that depends on the signal power is the PER $P_{u}(\mathbf{A})$. So, in order to maximize the goodput it is sufficient to find the set of values for the power coefficients $\left\{p^{(n)^{*}}\right\}_{n=1}^{N}$ that minimizes the PEP for each value of the Hamming distance $d$. This section outlines the structure of an iterative procedure that minimizes the PER upper bound given in (14), which will be addressed to as Goodput-Oriented Power Allocation policy (GOPA). First of all, recalling (5), (10) and (11), let rewrite the PEP as a function of the power allocation:

$$
P E P \leq\left[\sum_{n=1}^{N} \operatorname{Pr}(n) \exp \left(-p^{(n)} / \gamma^{(n)}\right)\right]^{d},
$$

where

$$
\gamma^{(n)} \triangleq \frac{4}{S\left|H^{(n)}\right|^{2} d_{\min }^{(n)^{2}}}
$$

and denote the vector containing the power allocated on the subcarriers with $\mathbf{p} \triangleq\left[p^{(1)}, \cdots, p^{(N)}\right]^{T}$. Because the monotonicity of the PEP with respect to $\underline{\mathbf{1}}^{T} \cdot \mathbf{p}$ we can solve the equivalent optimization problem given by

$$
\begin{array}{cc}
\underset{\underline{\mathbf{p}}}{\operatorname{minimize}} & F(\underline{\mathbf{p}}) \\
\text { subject to } & \underline{\mathbf{1}}^{T} \cdot \underline{\mathbf{p}} \leq N
\end{array}
$$

where

$$
F(\underline{\mathbf{p}}) \triangleq d \log \sum_{n=1}^{N} \operatorname{Pr}(n) \exp \left(-p^{(n)} / \gamma^{(n)}\right)
$$


is the natural logarithm of the PEP. Exploiting the CauchySchwarz inequality, can be easily demonstrated that

$$
\underline{\mathbf{v}}^{T}\left(\nabla^{2} F(\underline{\mathbf{p}})\right) \underline{\mathbf{v}}>0
$$

for each real vector $\underline{\mathbf{v}}$ when $p^{(n)}>0, \forall n$, where $\nabla^{2} F(\underline{\mathbf{p}})$ is the Hessian of the objective function. Consequently, $F(\underline{\mathbf{p}})$ is strictly convex over the set of feasible power allocations. This means that the optimization problem belongs to the class of convex optimization problem, where a convex objective function is to be minimized subject to a convex constraint set. According to the convex optimization theory, there is a unique vector $\underline{\mathbf{p}}^{*}$ such that

$$
F\left(\underline{\mathbf{p}}^{*}\right)<F(\underline{\mathbf{p}}) .
$$

In other words every local optimum is also a global optimum or, equivalently, the derivative of $F(\mathbf{p})$ from the optimal power allocation $\underline{\mathbf{p}}^{*}$ to any other point is positive:

$$
\left.\frac{d}{d \eta} F\left(\eta \underline{\mathbf{p}}+(1-\eta) \underline{\mathbf{p}}^{*}\right)\right|_{\eta=0} \geq 0 .
$$

The inequality (23) can be re-written as [5]

$$
\left.\frac{1}{N} \sum_{i=1}^{N}\left(p^{(i)}-p^{(i)^{*}}\right) \frac{\partial}{\partial p^{(i)}} F(\underline{\mathbf{p}})\right|_{p^{(i)}=p^{(i) *}} \geq 0
$$

Since the left-hand side of (24) is affine on $\mathbf{p}$, the optimality condition can be imposed only to the $N$ extreme values of the possible power allocations $\left\{\underline{\hat{\mathbf{p}}}_{i}\right\}_{i=0}^{N}$ so that the $i$ th point is defined as $p^{(i)}=N$ and $p^{(k)}=0, \forall k \neq i$. Substituting into the inequality we get

$$
\left.\frac{\partial}{\partial p^{(i)}} F(\underline{\mathbf{p}})\right|_{p^{(i)}=p^{(i) *}} \geq\left.\frac{1}{N} \sum_{i=1}^{N} p^{(i)^{*}} \frac{\partial}{\partial p^{(i)}} F(\underline{\mathbf{p}})\right|_{p^{(i)}=p^{(i) *}} .
$$

Moreover, when $p^{(n)}>0 \forall n$, the directional derivative in (23) vanishes and (24) becomes a strict equality. Let us remark that at the optimal point the value of the partial derivative of $F(\mathbf{p})$ with respect to $p^{(n)}$ doesn't depend on $n$. Roughly speaking, when $p^{(n)^{*}}>0 \forall n$, the derivative with respect to the power allocated over all subcarriers at the optimal point must be a constant value. Bearing in mind what discussed so far, an heuristic method to allocate the power among the subcarriers is outlined hereafter.

\section{B. Power Allocation Algorithm}

First of all, we take the derivative of the PEP natural logarithm, with respect to the coefficient $p^{(n)}$ of the power allocated on the generic $n$th subcarrier

$$
\begin{gathered}
\frac{\partial}{\partial p^{(n)}} F(\underline{\mathbf{p}})=-d \frac{\operatorname{Pr}(n)}{\gamma^{(n)}} \exp \left(-\frac{p^{(n)}}{\gamma^{(n)}}\right) \\
\frac{1}{\sum_{n=1}^{N} \operatorname{Pr}(n) \exp \left(-\frac{p^{(n)}}{\gamma^{(n)}}\right)}
\end{gathered}
$$

Starting with all the channels switched off, i.e. $p^{(n)}=0$, the derivative (26) is given by

$$
\frac{\partial}{\partial p^{(n)}} F(\underline{\mathbf{p}})=-d \frac{\operatorname{Pr}(n)}{\gamma^{(n)}} \quad \forall n .
$$

The power allocation algorithm can be summarized as follows:

- initialize $p^{(n)}=0, \forall i$. Sort the absolute values of the derivative (27) in the decreasing order and denote the corresponding subcarrier indexes by the vector $\left[n_{1}, \cdots, n_{N}\right]$. Then, increment the power $p^{\left(n_{1}\right)}$ until

$$
\begin{gathered}
\left.\frac{\partial}{\partial p^{\left(n_{1}\right)}} F(\underline{\mathbf{p}})\right|_{p^{\left(n_{1}\right)}=p_{1}^{\left(n_{1}\right)}}= \\
\left.\frac{\partial}{\partial p^{\left(n_{2}\right)}} F(\underline{\mathbf{p}})\right|_{p^{\left(n_{2}\right)}=0},
\end{gathered}
$$

when $p_{1}^{\left(n_{1}\right)}$ indicates the power increment for the first step. From (28) we get

$$
p_{1}^{\left(n_{1}\right)}=\gamma^{\left(n_{1}\right)} \log \left(\frac{\operatorname{Pr}\left(n_{1}\right) \gamma^{\left(n_{2}\right)}}{\operatorname{Pr}\left(n_{2}\right) \gamma^{\left(n_{1}\right)}}\right) .
$$

- In the second step, increment the power of both subcarriers $n_{1}$ and $n_{2}$, imposing that

$$
\begin{gathered}
\left.\frac{\partial}{\partial p^{\left(n_{1}\right)}} F(\underline{\mathbf{p}})\right|_{p^{\left(n_{1}\right)}=p_{1}^{\left(n_{1}\right)}+p_{2}^{\left(n_{1}\right)}}= \\
\left.\frac{\partial}{\partial p^{\left(n_{2}\right)}} F(\underline{\mathbf{p}})\right|_{p^{\left(n_{2}\right)}=p_{2}^{\left(n_{2}\right)}} .
\end{gathered}
$$

Substituting (26) in (30) and after some manipulation, we obtain

$$
\left|H^{\left(n_{1}\right)}\right|^{2} d_{\min }^{\left(n_{1}\right)^{2}} p_{2}^{\left(n_{1}\right)}=\left|H^{\left(n_{2}\right)}\right|^{2} d_{\min }^{\left(n_{2}\right)^{2}} p_{2}^{\left(n_{2}\right)} .
$$

We remark that equation (31) means that the power allocated at the second step yields uniform SNR increment. In other words, the SNR on either subcarrier has to be incremented by the same quantity. This result is obtained for each step and for each subcarrier.

- The power gain of $n_{1}$ and $n_{2}$ is incremented until their derivative is the same as the derivative on $n_{3}$. From this condition we can get the power allocation at the second step, and so on.

Generalizing at the $k$ th step the power expression and using the power constraint

$$
\begin{gathered}
\frac{1}{N}\left[p_{1}^{\left(n_{1}\right)}+p_{2}^{\left(n_{1}\right)}+\cdots+p_{k}^{\left(n_{1}\right)}+p_{2}^{\left(n_{2}\right)}+\cdots\right. \\
\left.\cdots+p_{k}^{\left(n_{k-1}\right)}+p_{k}^{\left(n_{k}\right)}\right]=1
\end{gathered}
$$

we obtain the optimum power allocation policy given by

$$
p^{(n)^{*}}=\frac{N-\sum_{\nu=1}^{N^{\prime}} \gamma^{(\nu)}\left[\log \frac{\operatorname{Pr}(\nu)}{\gamma^{(\nu)}}-\log \frac{\operatorname{Pr}(n)}{\gamma^{(n)}}\right]}{N^{\prime} \frac{\bar{\gamma}}{\gamma^{(n)}}},
$$

where $N^{\prime}$ is the number of subcarriers with a non-null power allocation and

$$
\bar{\gamma} \triangleq \frac{1}{N^{\prime}} \sum_{\nu=1}^{N^{\prime}} \gamma^{(\nu)}
$$




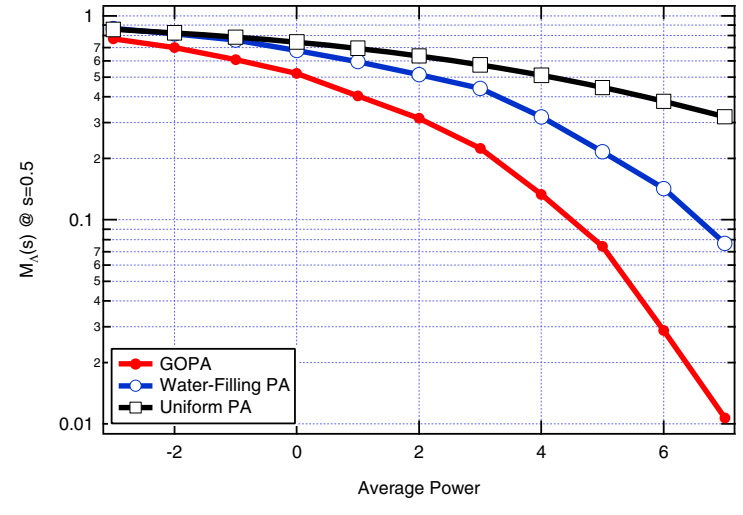

Fig. 2. MGF Vs Average Subcarrier Power.

is the average of $\gamma^{(\nu)}$ over the useful (active) subcarriers. Finally, substituting the GOPA solution into the condition (25), its optimality is easily demonstrated.

\section{Simulation RESUlts}

The effectiveness of the proposed power allocation policy in improving the goodput performance of a BICM-OFDM system in the presence of frequency selective channel is verified through numerical simulation. The performance of the proposed power allocation is also compared to the non adaptive scheme and the classical "water-filling" power allocation.

\section{A. System setup}

In the sequel, we will focus on a BICM-OFDM scheme with $N=64, N_{c p}=16$ and sample interval $T_{s}=50 \mathrm{~ns}$. The system operates with code rate $R=1 / 2$ and QPSK modulation for all the active subcarriers $\left(m^{(n)}=2, \forall n\right)$. The resulting bit rate is thus $R_{b}=N m^{(n)} R /\left(T_{s} N\right)=20 \mathrm{Mbit} / \mathrm{s}$. The signal experiences a 6 -tap multipath channel, wherein each path is modeled as a Rayleigh channel independent from others and the maximum delay spread is $0.6 \mu \mathrm{s}$. We have assumed a data packet length of 1 kbit.

\section{B. Numerical Results}

Let us now evaluate the system performance improvement that can be achieved when the proposed power allocation policy is applied. The results depicted in Fig. 2 show the MGF function evaluated at the saddlepoint for a given channel realization and for different power allocation strategies. The curves refer to : $i$ ) uniform power distribution (squares); $i$ ) water-filling power allocation (triangles); iii) GOPA algorithm (circles). Recalling the definition of the Battacharyya bound (10), we note that these curves are effective in guaranteing a better minimum PER performance with respect to the others strategies considered (and then in terms of Goodput when the same bit allocation strategy is assumed). The test shown in Fig. 3 deals with the goodput performance. The GOPA algorithm is shown to achieve a maximum gain of $16 \mathrm{bit} / \mathrm{OFDM}$ symbol with respect to the uniform power allocation and 4bit/OFDM symbol with respect to the water-filling.

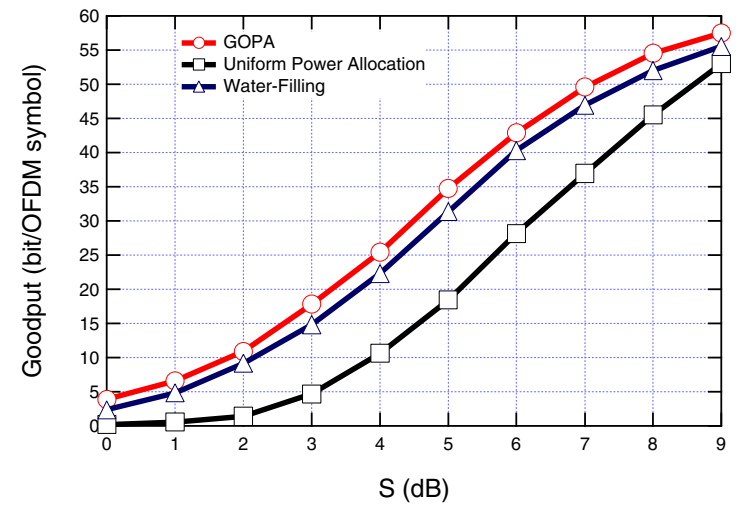

Fig. 3. Goodput Vs Average Subcarrier Power.

\section{CONCLUDing REMARKS}

A theoretical goodput analysis for the BICM-OFDM system under frequency selective fading channel has been presented. Based on this, we derived a power allocation policy to maximize the goodput performance and the optimality of this solution has been analytically demonstrated. The proposed approach has been verified by computer simulations under typical operating conditions. Numerical results have shown the effectiveness of the proposed strategy. The simulations show a goodput improvement with respect to the non adaptive case and to the "classical water filling" policy.

\section{ACKNOWLEDGMENT}

This work was supported by the European Commission in the framework of the FP7 Network of Excellence in Wireless COMmunications NEWCOM++ (contract n.216715)

\section{REFERENCES}

[1] E. Zehavi, 8-PSK Trellis Codes for a Rayleigh Channel, IEEE Trans. on Commun., Vol. 40, pp. 873-884, May 1992.

[2] G. Caire, G. Taricco, E. Biglieri, Bit-Interleaved Coded Modulation, IEEE Trans. on Inf. Theory, Vol. 44, pp. 927-946, May 1998.

[3] A. Martinez, A.G. Fabregas, G.Caire, Error Probability Analysis of BitInterleaved Coded Modulation, IEEE Trans. on Inf. Theory, Vol. 52, pp. 262-271, January 2006.

[4] K.-B. Song, A. Ekbal, S. T. Chung, Adaptive Modulation and Coding (AMC) for Bit-Interleaved Coded OFDM (BIC-OFDM), IEEE Trans. on Wireless Comm., Vol. 5, pp. 1685-1694, July 2006.

[5] A. Lozano, A. Tulino, S. Verdu, Optimum Power Allocation for Parallel Gaussian Channels With Arbitrary Input Distributions, IEEE Trans. on Inf. Theory, Vol. 52, NO. 7, pp. 3033-3051, July 2006.

[6] S. Liu, S. Zhou, G.B. Giannakis, Cross-Layer Combining of Adaptive Modulation and Coding with Truncated ARQ over Wireless Link, IEEE Trans. on Wireless Comm., Vol. 3, pp. 1746-1755, Sep. 2004.

[7] S. Lin, D. Costello, M.J. Miller, Automatic-Repeat-Request Error-Control Schemes, IEEE Comm. Mag., Vol. 22, pp 5-17, Dec. 1984.

[8] B. Devillers, L. Vandendorpe, Bit and Power Allocation for Goodput Optimization in Coded OFDM Systems, Proc. ICASSP 2006, Vol.4, pp 649-652, Toulouse, May 2006.

[9] R. McKay et Al. A Throughput-Based Adaptive MIMO-BICM Approach for Spatially-Correlated Channels, ICC 2006, June 2006.

[10] I. Stupia, L. Vandendorpe, J. Louveaux, F. Giannetti, V. Lottici, ,A.N. D'Andrea. Power Allocation for Goodput Optimization in BICM-OFDM Systems, ICC 2008, May 2008.

[11] T.M. Cover, J.A. Thomas, Elements of Information Theory, New York: Wiley, 1991.

[12] S. Boyd, L. Vandenberghe, Convex Optimization, Cambridge University Press, 2004. 